\title{
Distinctive Phyllosphere bacterial communities in tropical trees.
}

\begin{abstract}
Recent work has suggested that in temperate and subtropical trees, leaf surface bacterial communities are distinctive to each individual tree species and dominated by Alpha and Gammaproteobacteria. In order to understand how general this pattern is, we studied the phyllosphere bacterial community on leaves of six species of tropical trees at a rainforest arboretum in Malaysia. This represents the first detailed study of 'true' tropical lowland tree phyllosphere communities. Leaf surface DNA was extracted and pyrosequenced targeting the V1-V3 region of $16 \mathrm{~S}$ rRNA gene. As was previously found in temperate and subtropical trees, each tree species had a distinctive bacterial community on its leaves, clustering separately from other tree species in an ordination analysis. Bacterial communities in the phyllosphere were unique to plant leaves in that very few operational taxonomic units $(0.5 \%)$ co-occurred in the surrounding soil environment. A novel and distinctive aspect of tropical phyllosphere communities is that Acidobacteria were one of the most abundant phyla across all samples (on average, 17\%), a pattern not previously recognized. Sequences belonging to Acidobacteria were classified into subgroups 1-6 among known 24 subdivisions, and subgroup $1(84 \%)$ was the most abundant group, followed by subgroup $3(15 \%)$. The high abundance of Acidobacteria on leaves of tropical trees indicates that there is a strong relationship between host plants and Acidobacteria in tropical rain forest, which needs to be investigated further. The similarity of phyllosphere bacterial communities amongst the tree species sampled shows a significant tendency to follow host plant phylogeny, with more similar communities on more closely related hosts.
\end{abstract}

Keyword: Bacterial communities; tropical trees; Microbiology; Molecular genetics. 